\title{
Relationship between CHA2DS2-VASc and CHADS2 scores with pulmonary hypertension in patients with acute pulmonary embolism
}

\begin{abstract}
Introduction: Pulmonary hypertension (PH) is the most important prognostic factor after acute pulmonary embolism (PE). Therefore, determination of patients who will develop $\mathrm{PH}$ after acute $\mathrm{PE}$ is crucial. The aim of the present study was to evaluate the predictive value of the CHADS2 and CHA2DS2-VASc scores for PH in patients with acute PE.

Material and methods: Seventy-nine adults who presented with acute PE, had an admission systolic pulmonary artery pressure (sPAP) measured on echocardiogram and no previous history of $\mathrm{PE}$, were retrospectively identified from the computerized database. 31 patients who had SPAP $\leq 40 \mathrm{~mm} \mathrm{Hg}$ were categorized as a "normal pulmonary pressure" group, whereas 48 patients who had sPAP > $40 \mathrm{~mm} \mathrm{Hg}$ were categorized as a "PH" group.

Results: SPAP was $>40 \mathrm{~mm} \mathrm{Hg}$ in 48 patients $(60.8 \%$ ), with a mean sPAP of $60.9 \pm 16.1 \mathrm{~mm} \mathrm{Hg}$ (median $=60, \min -\max =$ 41-100 mm Hg). In multivariate logistic regression models adjusted for CHADS2 and CHA2DS2-VASc score components, only age was found to be related with the development of PH. SPAP was weakly positively correlated with CHADS2 $(p=0.047 ; r=$ $0.224)$ and CHA2DS2-VASc $(p=0.023 ; r=0.256)$ scores. SPAP values were increasing with the severity of the scores.

Conclusions: Both CHADS2 and CHA2DS2-VASc scores could be useful in the determination of which patients should be closely followed up in order to prevent the development of PH after acute PE.
\end{abstract}

Key words: CHA2DS2-VASc score, CHADS2 score, pulmonary hypertension, acute pulmonary embolism

Adv Respir Med. 2019; 87: 203-208

\section{Introduction}

Acute pulmonary embolism (PE) is one of the major causes of mortality, morbidity, and hospitalization worldwide [1]. Since patients older than 40 years are at an increased risk compared with younger patients, and the risk approximately doubles with each subsequent decade, an ever-larger number of patients are expected to be diagnosed with PE in the future [2]. The most important short- and long-term complication of $\mathrm{PE}$ is pulmonary hypertension $(\mathrm{PH})$. The occurrence of $\mathrm{PH}$ after acute $\mathrm{PE}$ is strongly related with prognosis [3]. Hence, the risk stratification of PE to determine the development of $\mathrm{PH}$ is crucial.
The $\mathrm{CHA}_{2} \mathrm{DS}_{2}$-VASc (congestive heart failure, hypertension, age $\geq 75$ years, diabetes mellitus, previous stroke or transient ischemic attack (TIA), vascular disease, age 65 to 74 years, female gender) score and $\mathrm{CHADS}_{2}$ (congestive heart failure, hypertension, age $\geq 75$ years, diabetes mellitus, previous stroke or TIA) scores are used for embolic risk stratification in patients with atrial fibrillation (AF) [4]. Recent studies have demonstrated that both scores can predict prognosis in subjects with stable coronary artery disease, acute coronary syndrome and coronary artery bypass grafting surgery, irrespective of the presence of AF [5-7].

The aim of the present study is to evaluate the relation of CHA2DS2-VASc and CHADS2

Address for correspondence: Samet Yilmaz, Pamukkale University Hospitals, Denizli, Turkey; e-mail: sametyilmazmd@gmail.com

DOI: 10.5603/ARM.2019.0057

Received: 11.06 .2019

Copyright (C) 2019 PTChP

ISSN 2451-4934 
scores with the development of $\mathrm{PH}$ in patients with acute PE.

\section{Material and methods}

In this retrospective study, 79 patients with diagnoses of $\mathrm{PE}$ were consecutively selected from hospital database. The patients' demographic, laboratory and echocardiographic data were obtained from hospital records. The study was in compliance with the principles outlined in the Declaration of Helsinki and approved by the local ethics committee.

Diagnosis of PE was made by an emergency department physician, cardiologist and chest disease consultant. Thoracal pulmonary computed tomography angiography (CTA) was performed to all the patients. Admission symptoms, hemodynamic profile, electrocardiographic features and echocardiographic findings were obtained from hospital records. The patients' clinical and demographic characteristics, encompassing age, gender, history of arterial hypertension, diabetes mellitus, tobacco use, left ventricular (LV) ejection fraction and systolic pulmonary arterial pressure measurement (sPAP) were noted.

The estimation of sPAP was calculated by the peak tricuspid regurgitation velocity (TRV) taking into account right atrial pressure (RAP) as described by the simplified Bernoulli equation [8]. RAP was estimated by echocardiography based on the diameter and respiratory variation in diameter of the inferior vena cava (IVC): an IVC diameter $<2.1 \mathrm{~cm}$ that collapses $>50 \%$ with a sniff suggested a normal RAP of $3 \mathrm{~mm}$

Table 1. Basic demographic, clinical, laboratory and echocardiographic features of the study population

\begin{tabular}{|c|c|c|c|c|c|}
\hline \multirow[b]{2}{*}{ Age } & \multicolumn{2}{|c|}{$\begin{array}{l}\text { Normal pulmonary pressure group } \\
\qquad(\mathrm{n}=\mathbf{3 1})\end{array}$} & \multicolumn{2}{|c|}{$\begin{array}{l}\text { Pulmonary hypertension group } \\
\qquad(\mathrm{n}=48)\end{array}$} & \multirow{2}{*}{$\begin{array}{c}\mathbf{P} \\
0.007^{*}\end{array}$} \\
\hline & $60.2 \pm 17.3$ & $64(22-93)$ & $69.9 \pm 14.2$ & $74(29-88)$ & \\
\hline Male sex & 18 & $58.10 \%$ & 25 & $52.10 \%$ & 0.602 \\
\hline Congestive heart failure & 3 & $9.70 \%$ & 7 & $14.60 \%$ & 0.732 \\
\hline Diabetes & 11 & $35.50 \%$ & 22 & $45.80 \%$ & 0.362 \\
\hline Hypertension & 18 & $58.10 \%$ & 28 & $58.30 \%$ & 0.981 \\
\hline Coronary artery disease & 17 & $54.80 \%$ & 17 & $35.40 \%$ & 0.089 \\
\hline Peripheral artery disease & 2 & $6.50 \%$ & 5 & $10.40 \%$ & 0.698 \\
\hline Stroke or TIA history & 1 & $3.20 \%$ & 9 & $18.80 \%$ & 0.079 \\
\hline Hiperlipidemi & 10 & $32.30 \%$ & 13 & $27.10 \%$ & 0.621 \\
\hline Glucose (mg/dL) & $109.6 \pm 34.4$ & $98.5(62-196)$ & $116.6 \pm 36.5$ & $106(69-215)$ & 0.27 \\
\hline Creatinine (mg/dL) & $0.9 \pm 0.2$ & $0.9(0.4-1.4)$ & $1.1 \pm 0.5$ & $0.9(0.6-4.1)$ & 0.481 \\
\hline Uric acid (mg/dL) & $5.5 \pm 2$ & $5.4(2.2-9.2)$ & $7.7 \pm 2.8$ & $6.3(4.6-15.7)$ & $0.01^{*}$ \\
\hline Hemoglobin $(\mathrm{g} / \mathrm{dL})$ & $12.5 \pm 2$ & $12.2(8.7-15.8)$ & $13.7 \pm 11.4$ & $12.1(7.1-88.9)$ & 0.418 \\
\hline Platelet count $\left(10^{3} / \mathrm{mL}\right)$ & $255.1 \pm 84$ & $250(94-450)$ & $237 \pm 89.6$ & $232(57-419)$ & 0.381 \\
\hline White blood cell count $\left(10^{3} / \mathrm{mL}\right)$ & $7.8 \pm 2.3$ & $7.5(3.4-14.3)$ & $8.2 \pm 2.9$ & $7.6(3.7-19)$ & 0.77 \\
\hline Troponin I (ng/mL) & $1.3 \pm 2.7$ & $0.2(0-8.7)$ & $0.5 \pm 0.6$ & $0.2(0-2.7)$ & 0.797 \\
\hline CK-MB (mg/L) & $24.9 \pm 13.7$ & $31(0.2-40)$ & $28.1 \pm 10.1$ & $28(0.3-48)$ & 0.969 \\
\hline D-dimer (mcg/L) & $5.2 \pm 4.7$ & $4.4(1-24.1)$ & $5.9 \pm 5.6$ & $4.3(0-23)$ & 0.969 \\
\hline$C$ reactive protein & $36.9 \pm 33.3$ & $17.2(4-96)$ & $34.6 \pm 29.3$ & $26.1(0.1-132)$ & 0.728 \\
\hline LV ejection fraction (\%) & $55.9 \pm 8$ & $60(30-66)$ & $50.3 \pm 12.1$ & $55(20-65)$ & $0.008^{*}$ \\
\hline SPAP $(m m ~ H g)$ & $34.3 \pm 4.2$ & $34(28-40)$ & $60.9 \pm 16.1$ & $60(41-100)$ & $0.0001^{*}$ \\
\hline $\mathrm{CHADS}_{2}$ score & $1.3 \pm 1.2$ & $1(0-5)$ & $2 \pm 1.4$ & $2(0-5)$ & $0.024^{*}$ \\
\hline $\mathrm{CHA}_{2} \mathrm{DS}_{2}$-VASc score & $2.6 \pm 1.9$ & $2(1-8)$ & $3.5 \pm 1.9$ & $3.5(0-7)$ & $0.015^{*}$ \\
\hline
\end{tabular}

Data are presented as mean \pm std deviation, median (min-max values) or number (percentage). ${ }^{*} \mathrm{p}<0.05$ statistically significant; Independent Samples T test; MannWhitney U test; Chi Square Test, $\mathrm{CHA}_{2} \mathrm{DS}_{2}$-VASc - congestive heart failure, hypertension, age $\geq 75$ years, diabetes mellitus, previous stroke or transient ischemic attack, vascular disease, age 65 to 74 years, female gender; $\mathrm{CHADS}_{2}$ - congestive heart failure, hypertension, age $\geq 75$ years, diabetes mellitus, previous stroke or transient ischemic attack; CK-MB — creatinine kinase myoglobin isoform; DVT — deep venous thrombosis; LV — left ventricular; SPAP — systolic pulmonary artery pressure; TIA — transient ischemic attack 
Table 2. Pulmonary embolism risk factors

\begin{tabular}{lccc}
\hline & $\begin{array}{c}\text { Normal pulmonary pressure group } \\
(\mathbf{n}=\mathbf{3 1})\end{array}$ & $\begin{array}{c}\text { Pulmonary hypertension group } \\
(\mathbf{n}=\mathbf{4 8})\end{array}$ & $\begin{array}{c}\text { P } \\
\text { Malignancy } \mathrm{n}(\%)\end{array}$ \\
Immobilization $\mathrm{n}(\%)$ & $3(9.7)$ & $4(8.3)$ & 1.000 \\
Surgery n (\%) & $4(12.9)$ & $13(27)$ & 0.134 \\
DVT history $\mathrm{n}(\%)$ & $7(22.5)$ & $7(14.5)$ & 0.363 \\
Pregnancy $\mathrm{n}(\%)$ & $4(12.9)$ & $1(2.1)$ & 0.075 \\
Travel history $\mathrm{n}(\%)$ & $2(6.5)$ & 0 & 0.151 \\
\hline
\end{tabular}

DVT — deep venous thrombosis; immobilization — immobilization $\geq 3$ days; surgery — surgery in the last month; travel history — travel history $>6$ hours in the past week

$\mathrm{Hg}$ (range 0-5 $\mathrm{mm} \mathrm{Hg}$ ), whereas an IVC diameter $>2.1 \mathrm{~cm}$ that collapses $<50 \%$ with a sniff or on quiet inspiration suggested a high RAP of $15 \mathrm{~mm} \mathrm{Hg}$ (range $10-20 \mathrm{~mm} \mathrm{Hg}$ ). In patients in whom the IVC diameter and collapse did not fit this paradigm, an intermediate value of $8 \mathrm{~mm} \mathrm{Hg}$ (range $5-10 \mathrm{~mm} \mathrm{Hg}$ ) was used. PH was defined as an increase in mean pulmonary arterial pressure (mPAP) $\geq 25 \mathrm{~mm} \mathrm{Hg}$, and a sPAP of $40 \mathrm{~mm} \mathrm{Hg}$ typically implies a mPAP more than $25 \mathrm{~mm} \mathrm{Hg}$ [8].

On the basis of the $\mathrm{CHA}_{2} \mathrm{DS}_{2}$-VASc score, patients were assigned 1 point for congestive heart failure, hypertension, age 65-74 years, diabetes mellitus, vascular disease, female sex; 2 points for age 75 years or older and previous stroke or TIA. $\mathrm{CHADS}_{2}$ score was calculated by the sum of 1 point for each congestive heart failure, hypertension, age $\geq 75$ years, diabetes mellitus and 2 points for previous stroke or TIA.

Data analysis was performed using SPSS Statistics for Windows, version 24.0 (SPSS, Inc., Chicago, IL, USA). Kolmogorov-Smirnov and Shapiro-Wilk tests were used to examine distribution pattern. Data were presented as mean \pm standard deviation, median (minimum-maximum values) for continuous variables. The number of cases and percentages were used for categorical data. Independent Samples T test was applied for comparisons of data that were normally distributed; otherwise the Mann-Whitney U test was applied. Categorical data were analyzed using the Chi Square test. The effect of each different variable on the development of $\mathrm{PH}$ was calculated in univariate and multivariate analysis. Receiver operating characteristics (ROC) curve analysis was performed to find the best cut-off points of $\mathrm{CHADS}_{2}$ and $\mathrm{CHA}_{2} \mathrm{DS}_{2}$-VASc scores for the development of $\mathrm{PH}$. P-value $<0.05$ was considered statistically significant.

\section{Results}

A total of 79 patients (mean age: 66.1 \pm 16.1 years, $54.4 \%$ men) were included in the study. Patients were grouped according to sPAP values on admission. Thirty-one patients who had sPAP $\leq 40 \mathrm{~mm} \mathrm{Hg}$ were categorized as the "normal pulmonary pressure" group, whereas 48 patients (60.8\%) who had sPAP > $40 \mathrm{~mm} \mathrm{Hg}$ were categorized as the "PH" group.

Basic demographic, clinical and laboratory parameters of patients are presented in Table 1. SPAP value of the normal pulmonary pressure group was significantly lower than the pulmonary hypertension group $(\mathrm{p}<0.0001)$. CHADS2 and CHA2DS2-VASc scores were significantly higher in the $\mathrm{PH}$ group $(\mathrm{p}=0.024 ; \mathrm{p}=0.015)$. There was no significant difference in terms of PE risk factors, including malignancy, surgery, immobilization, pregnancy, deep venous thrombosis and travel history (Table 2).

According to univariate logistic regression analysis, CHADS2 score [odds ratio (OR): 1.538, 95\% CI 1.043-2.268, $\mathrm{p}=0.030]$, CHA2DS2-VASc score (OR: 1.318, 95\% CI 1.016-1.711, $\mathrm{p}=0.038$ ), age (OR: 1.041, 95\% CI 1.009-1.073, $\mathrm{p}=0.012$ ) and LV ejection fraction (OR: 0.939, 95\% CI 0.885-0.997, $\mathrm{p}=0.038$ ) were found to be related to $\mathrm{PH}$ (Table 3).

In multivariate logistic regression models adjusted for CHADS2 and CHA2DS2-VASc score components, only age was found to be related with the development of PH (OR: 1.037, p $=0.030$ in model 1; OR: 1.041, $\mathrm{p}=0.024$ in model 2).

ROC curve analysis for CHADS2 and CHA2DS2-VASc scores and PH were shown in Figure 1 and 2. The optimal cut-off value of CHADS2 and CHA2DS2-VASc scores for predicting the development of $\mathrm{PH}$ were 1.5 and 2.5, respectively in ROC curve analysis. Any CHADS2 value greater 
Table 3. Univariate and multivariate logistic regression analyses to predict pulmonary hypertension

\begin{tabular}{|c|c|c|c|}
\hline Univariate models & $\begin{array}{l}\text { Odds } \\
\text { ratio }\end{array}$ & $\begin{array}{l}95 \% \text { Confiden- } \\
\text { ce interval }\end{array}$ & $\mathbf{P}$ \\
\hline Age & 1.041 & $1.009-1.073$ & 0.012 \\
\hline Gender (male) & 1.274 & $0.512-3.167$ & 0.602 \\
\hline Coronary artery disease & 0.452 & $0.180-1.136$ & 0.091 \\
\hline Congestive heart failure & 1.593 & $0.379-6.694$ & 0.525 \\
\hline Hypertension & 1.011 & $0.405-2.526$ & 0.981 \\
\hline Diabetes mellitus & 1.538 & $0.607-3.897$ & 0.364 \\
\hline Peripheral artery disease & 1.686 & $0.306-9.286$ & 0.548 \\
\hline D-dimer (mcg/L) & 1.027 & $0.915-1.152$ & 0.656 \\
\hline $\begin{array}{l}\text { Chronic obstructive pul- } \\
\text { monary disease }\end{array}$ & 0.473 & $0.116-1.920$ & 0.295 \\
\hline LV ejection fraction (\%) & 0.939 & $0.885-0.997$ & 0.038 \\
\hline CHADS2 score & 1.538 & $1.043-2.268$ & 0.030 \\
\hline CHA2DS2-VASc score & 1.318 & $1.016-1.711$ & 0.038 \\
\hline \multicolumn{4}{|l|}{ Multivariate models } \\
\hline \multicolumn{4}{|l|}{ Model 1} \\
\hline Congestive heart failure & 1.041 & $0.219-4.959$ & 0.959 \\
\hline Hypertension & 1.668 & $0.598-4.653$ & 0.328 \\
\hline Age & 1.037 & $1.004-1.071$ & 0.030 \\
\hline Diabetes mellitus & 0.687 & $0.249-1.892$ & 0.467 \\
\hline Stroke history & 0.185 & $0.020-1.683$ & 0.134 \\
\hline \multicolumn{4}{|l|}{ Model 2} \\
\hline Congestive heart failure & 1.637 & $0.305-8.777$ & 0.565 \\
\hline Hypertension & 1.455 & $0.494-4.280$ & 0.496 \\
\hline Age & 1.041 & $1.005-1.078$ & 0.024 \\
\hline Diabetes mellitus & 0.780 & $0.259-2.349$ & 0.659 \\
\hline Stroke history & 0.125 & $0.019-1.624$ & 0.125 \\
\hline Coronary artery disease & 2.889 & $0.976-8.551$ & 0.055 \\
\hline Female sex & 0.801 & $0.272-2.354$ & 0.686 \\
\hline
\end{tabular}

than 1.5 had a sensitivity of $58 \%$, a specificity of $58 \%$, and any CHA2DS2-VASc score greater than 2.5 had a sensitivity of $60 \%$ and a spesificity of $58 \%$ to predict the occurrence of $\mathrm{PH}$.

There was a weak positive correlation between sPAP and CHADS2 ( $\mathrm{p}=0.047 ; \mathrm{r}=0.224)$ and CHA2DS2-VASc $(\mathrm{p}=0.023 ; \mathrm{r}=0.256)$ scores (Figure 3-4). Increased sPAP values were associated with increased scores.

\section{Discussion}

Acute PE, which is an emergency condition, requires precise recognition and timely risk stratification, because early recognition and accurate

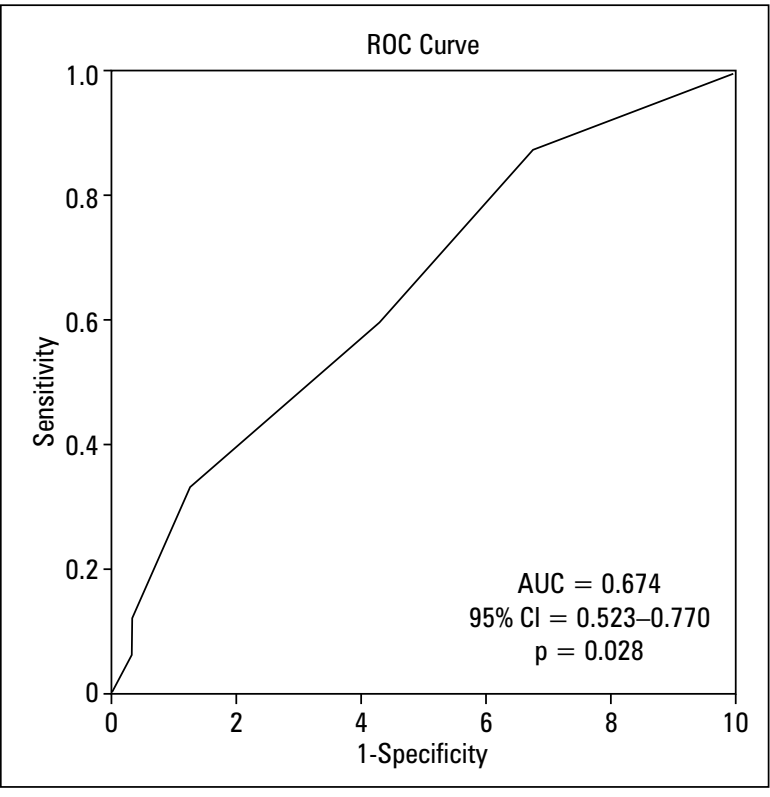

Figure 1. Receiver operating characteristic curve analyses of CHADS2 score for pulmonary hypertension

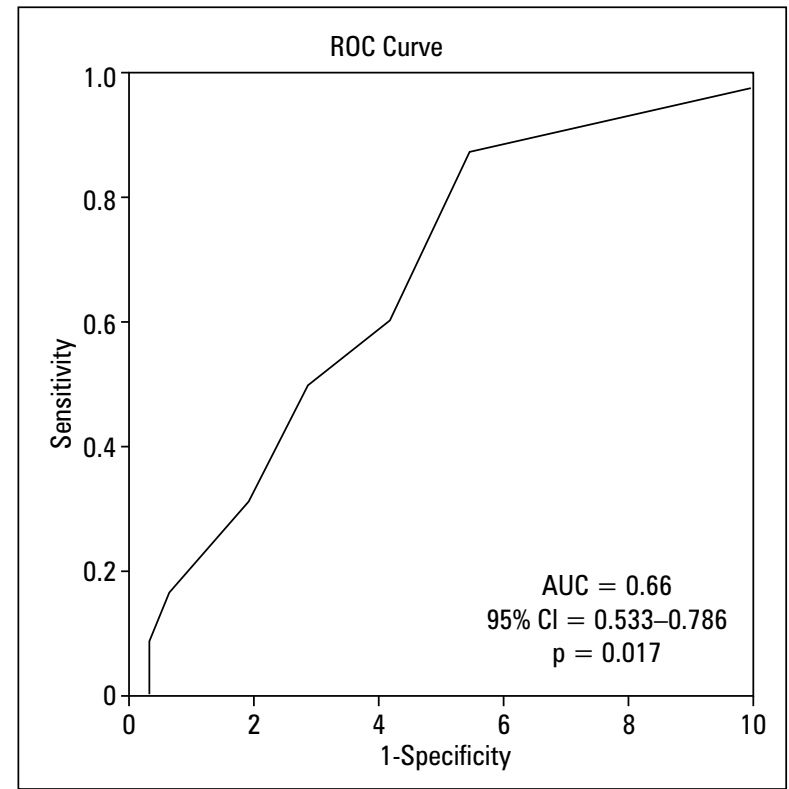

Figure 2. Receiver operating characteristic curve analyses of $\mathrm{CHA}_{2} \mathrm{DS}_{2}$ -VASc score for pulmonary hypertension

risk stratification determine prognosis [8]. Risk stratification in acute PE begins with initial hemodynamic status assessment at an emergency department, being a well-established marker of prognosis. It is followed by laboratory tests and echocardiographic evaluation. Large and multiple emboli abruptly increase pulmonary vascular resistance and cause $\mathrm{PH}$ which in turn causes right ventricular (RV) strain. An acute increase of pulmonary pressure is directly related to RV 


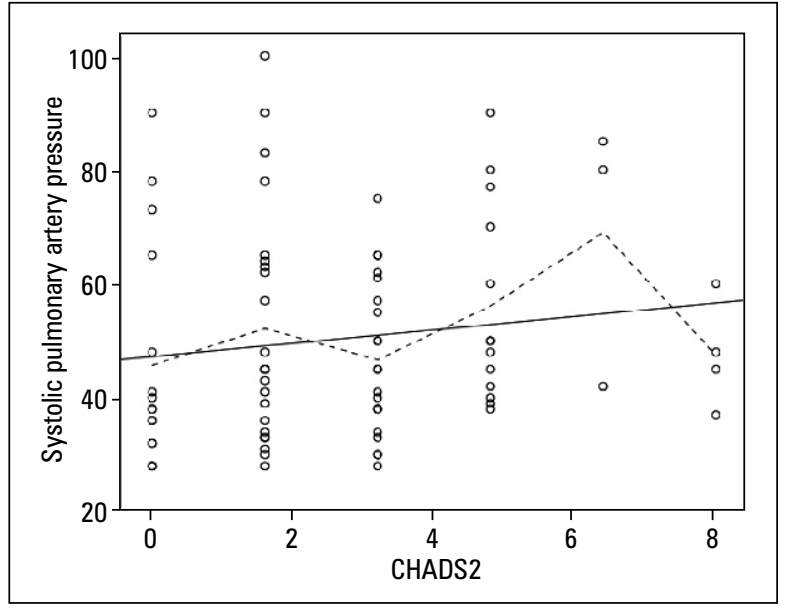

Figure 3. Correlation between systolic pulmonary artery pressure and $\mathrm{CHADS}_{2}$ score

myocardial damage, and it is connected with prognosis [9]. Because of these reasons, estimation of pulmonary pressure by invasive or non-invasive techniques is important. In the acute setting of PE, invasive pulmonary pressure analysis is not an easy one, therefore, echocardiographic evaluation is the cornerstone of the prognosis assessment.

Over the past decade, it has been documented that sPAP may help estimate mPAP in adults with high accuracy and reasonably good precision [10]. The $25 \mathrm{~mm}$ Hg threshold used to define PH could correspond to an sPAP of 38-40 mm Hg [11]. Although the limits of the echocardiographic estimation of sPAP are widely appreciated, the results from invasive studies support an evidence-based sPAP-derived mPAP value, which is currently used to diagnose and follow patients with $\mathrm{PH}$. As suggested by these researches, in this study, we determined the subjects with $\mathrm{PH}$ based on echocardiographic sPAP values over $40 \mathrm{~mm} \mathrm{Hg}$.

Chronic thromboembolic pulmonary hypertension (CTEPH) is a clinical entity with high pulmonary pressure that occurs after a thromboembolic event. More than $70 \%$ of CTEPH patients have an history of PE [12]. Therefore, individuals with PE should be screened routinely for the development of CTEPH.

CHADS2 and $\mathrm{CHA}_{2} \mathrm{DS}_{2}$-VASc scores are clinical thromboembolic risk scores for predicting stroke in patients from the high-risk population and with non-valvular AF [13]. However, recent studies have demonstrated that both scores can predict mortality in various cardiovascular diseases, irrespective of the presence of $\mathrm{AF}[14,15]$. In our study, the subjects with $\mathrm{PH}$ had significantly higher $\mathrm{CHA}_{2} \mathrm{DS}_{2}$-VASc and CHADS2 scores com-

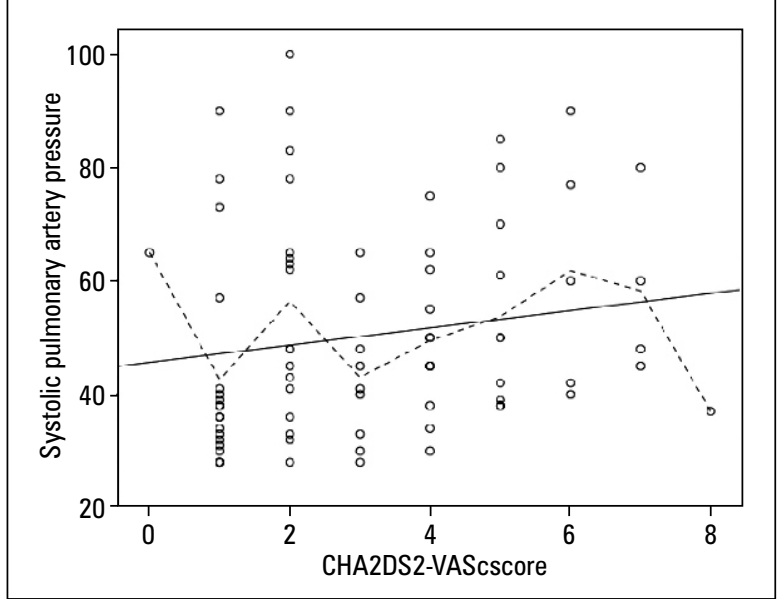

Figure 4. Correlation between systolic pulmonary artery pressure and $\mathrm{CHA}_{2} \mathrm{DS}_{2}$-VASc score

pared to the normal pulmonary pressure group. We found a positive correlation between sPAP and CHADS2 and CHA2DS2-VASc scores. Among $\mathrm{CHA}_{2} \mathrm{DS}_{2}$-VASc score components; age, hypertension, diabetes mellitus and the presence of vascular disease are well-known risk factors for deep venous thrombosis, which are also the risk factors for acute PE [16]. Therefore, these scores may be used to predict the development of $\mathrm{PE}$ and $\mathrm{PH}$.

Although there was a significant difference in CHADS2 and CHA2DS2-VASc scores between patients with $\mathrm{PH}$ and normal pulmonary pressure groups, only older age reached statistical significance in multivariate regression analysis. This result showed us that the older age is the strongest predictor of $\mathrm{PH}$ after acute $\mathrm{PE}$. Older age, especially above 80, is also one of the parameters of Pulmonary Embolism Severity Index (PESI), which is the most reliable and validated risk score system after acute PE [17]. The presence of chronic heart failure is also a parameter of CHADS2 and CHA2DS2-VASc scores, and it is also a component of a PESI index. The most recent 2019 European Society of Cardiology guidelines for the diagnosis and management of acute pulmonary embolism developed in collaboration with the European Respiratory Society recommends the determination of a clinical risk profile by using PESI index [17]. According to our current knowledge, our research is the first study using CHADS2 and CHA2DS2-VASc scores for risk stratification after acute PE.

On the other hand, the study has some limitations. First, it has a retrospective cross-sectional design with data from a single center. Due to the retrospective nature of our data and the small sample size, our findings can not be generalized 
to all populations. Second, pulmonary pressure estimation and determination of patients with $\mathrm{PH}$ were done with echocardiography. Invasive right heart catheterization was not performed. Finally, sPAP measurements were used instead of mPAP, which might have led to misdiagnosis of $\mathrm{PH}$.

\section{Conclusions}

In our study, older age is the most important factor related to the development of $\mathrm{PH}$ after acute PE. Both CHADS2 and CHA2DS2-VASc scores could be useful in acute PE setting in the determination of which patients should be closely followed up so that more prolonged anticoagulation therapy and surveillance concerning the development of PH may be applied.

\section{Conflict of interest}

The authors declare no conflict of interest.

\section{References:}

1. Konstantinides SV, Torbicki A, Agnelli G, et al. 2014 ESC guidelines on the diagnosis and management of acute pulmonary embolism. Eur Heart J. 2014; 35(43): 3033-3073, doi: 10.1093/eurheartj/ehu283, indexed in Pubmed: 25173341.

2. Anderson FA, Spencer FA. Risk factors for venous thromboembolism. Circulation. 2003; 107(23 Suppl 1): I9-16, doi: 10.1161/01.CIR.0000078469.07362.E6, indexed in Pubmed: 12814980.

3. Barco S, Klok FA, Konstantinides SV, et al. Chronic thromboembolic pulmonary hypertension. J Am Coll Cardiol. 2013; 62(25 Suppl): D92-D99, doi: 10.1016/j.jacc.2013.10.024, indexed in Pubmed: 24355646.

4. January CT, Wann LS, Calkins H, et al. 2014 AHA/ACC/HRS guideline for the management of patients with atrial fibrillation: a report of the American College of Cardiology/American Heart Association Task Force on practice guidelines and the Heart Rhythm Society. J Am Coll Cardiol. 2014; 64(21): e1e76, doi: 10.1161/CIR.0000000000000041, indexed in Pubmed: 24682347.

5. Orvin K, Bental T, Assali A, et al. Usefulness of the CHA2DS2-VASC score to predict adverse outcomes in patients having percutaneous coronary intervention. Am J Cardiol. 2016; 117(9): 1433-1438, doi: 10.1016/j.amjcard.2016.02.010, indexed in Pubmed: 27001448.

6. Huang SS, Chen YH, Chan WL, et al. Usefulness of the CHADS2 score for prognostic stratification of patients with acute myocardial infarction. Am J Cardiol. 2014; 114(9): 1309-1314, doi: 10.1016/j.amjcard.2014.07.063, indexed in Pubmed: 25205632 .
7. Lu DY, Huang CC, Huang PH, et al. Usefulness of the CHADS score for prognostic stratification in patients with coronary artery disease having coronary artery bypass grafting. Am J Cardiol. 2017; 119(6): 839-844, doi: 10.1016/j.amjcard.2016.11.035, indexed in Pubmed: 28057218.

8. Galiè N, Humbert M, Vachiery JL, et al. 2015 ESC/ERS guidelines for the diagnosis and treatment of pulmonary hypertension: the Joint Task Force for the Diagnosis and Treatment of Pulmonary Hypertension of the European Society of Cardiology (ESC) and the European Respiratory Society (ERS): endorsed by: Association for European Paediatric and Congenital Cardiology (AEPC), International Society for Heart and Lung Transplantation (ISHLT). Eur Heart J. 2016; 37(1): 67-119, doi: 10.1093/eurheartj/ehv317, indexed in Pubmed: 26320113.

9. Kurzyna M, Torbicki A, Pruszczyk P, et al. Disturbed right ventricular ejection pattern as a new Doppler echocardiographic sign of acute pulmonary embolism. Am J Cardiol. 2002; 90(5): 507-511, doi: 10.1016/s0002-9149(02)02523-7, indexed in Pubmed: 12208411.

10. Chemla D, Humbert M, Sitbon O, et al. Systolic and mean pulmonary artery pressures: are they interchangeable in patients with pulmonary hypertension? Chest. 2015; 147(4): 943-950, doi: 10.1378/chest.14-1755, indexed in Pubmed: 25846528.

11. Rudski LG. Point: can Doppler echocardiography estimates of pulmonary artery systolic pressures be relied upon to accurately make the diagnosis of pulmonary hypertension? Yes. Chest. 2013; 143(6): 1533-1536, doi: 10.1378/chest.13-0296, indexed in Pubmed: 23732579.

12. Pepke-Zaba J, Delcroix M, Lang I, et al. Chronic thromboembolic pulmonary hypertension (CTEPH): results from an international prospective registry. Circulation. 2011; 124(18): 1973-1981, doi: 10.1161/CIRCULATIONAHA.110.015008, indexed in Pubmed: 21969018.

13. Camm AJ, Lip GY, De Caterina R, et al. 2012 focused update of the ESC guidelines for the management of atrial fibrillation: an update of the 2010 ESC guidelines for the management of atrial fibrillation developed with the special contribution of the European Heart Rhythm Association. Eur Heart J. 2012; 33(21): 2719-2747, doi: 10.1093/eurheartj/ehs253, indexed in Pubmed: 22922413.

14. Huang FY, Huang BT, Pu Xb, et al. CHADS2, CHA2DS2-VASc and R2CHADS2 scores predict mortality in patients with coronary artery disease. Intern Emerg Med. 2017; 12(4): 479-486, doi: 10.1007/s11739-017-1608-x.

15. Hong C, Alluri K, Shariff N, et al. Usefulness of the CHA2DS2-VASC score to predict mortality in defibrillator recipients. Am J Cardiol. 2017; 120(1): 83-86, doi: 10.1016/j.amjcard.2017.03.257, indexed in Pubmed: 28479166.

16. Stone J, Hangge P, Albadawi $\mathrm{H}$, et al. Deep vein thrombosis: pathogenesis, diagnosis, and medical management. Cardiovasc Diagn Ther. 2017; 7(Suppl 3): S276-S284, doi: 10.21037/ cdt.2017.09.01, indexed in Pubmed: 29399531.

17. Konstantinides SV, Meyer G, Becattini C, et al. 2019 ESC Guidelines for the diagnosis and management of acute pulmonary embolism developed in collaboration with the European Respiratory Society (ERS): The Task Force for the diagnosis and management of acute pulmonary embolism of the European Society of Cardiology (ESC). Eur Heart J. 2019; 54(3): 1901647, doi: 10.1183/13993003.01647-2019, indexed in Pubmed: 31473594 